\title{
Joint Estimation-Segmentation of Optic Flow
}

\author{
Étienne Mémin ${ }^{1}$ and Patrick Pérez ${ }^{2}$ \\ 1 Valoria, Université de Bretagne Sud BP 1104, 56014 Vannes, France and \\ IRISA 35042 Rennes Cedex; memin@irisa.fr \\ ${ }^{2}$ IRISA/INRIA 35042 Rennes Cedex, France; perez@irisa.fr
}

\begin{abstract}
In this paper we address the intricate issue of jointly recovering the apparent velocity field between two consecutive frames and its underlying partition. We design a global cost functional including robust estimators. These estimators enable to deal with the large deviations occurring in the different energy terms and offer the possibility to introduce a simple coupling between a dense optical flow field and a segmentation. This coupling is also reinforced by a parametric likeness term. The resulting estimation-segmentation model thus involves a tight cooperation between a local estimation process and a global modelization. The minimization of the final cost function is conducted efficiently by a multigrid optimization algorithm.
\end{abstract}

\section{Introduction}

Motion estimation and motion-based segmentation are well known to be two tightly interwoven processes in motion analysis. It is obvious that a good estimation of the velocity field (or at least a sensible approximation of it) is required to obtain a good segmentation of the different apparent motions observable in the scene. At the opposite, a good velocity map cannot be obtained without an accurate estimation of the frontiers of the different moving objects. It is therefore natural to consider the resolution of these two motion problems as a whole.

The coupling of motion estimation and segmentation has been considered in various ways the last decade. Two classes of methods may be indeed distinguished. The first class consists in an unilateral coupling between some motion cues and a segmentation process. Methods belonging to this class assume that one of the set of variables is known in order to recover the second one. For instance, in [2] the optical flow is first estimated as a dense field and then fixed during all the segmentation process. Segmentation based on the normal flow field relies on the same philosophy $[1,3,7,17]$. The segmentation problem has been modeled within a number of frameworks: Markov random field theory [7, 17], statistical mixture estimation framework [3], minimum description length paradigm [10] or region split and merge methodology [2]. All these methods are based on a regionwise parametric description of the motion cues (i.e., the optical flow field or the normal flow field). More recently, coupled motion-based estimation-segmentation methods have been proposed. They involve a tight coupling between a motion field and a motion-based partition. In that case, the 
motion field and the associated segmentation map are estimated simultaneously. This is usually done by using a global energy function that ties both entities. In that context, different types of interaction have been proposed. In [18] for instance, the partition frontiers are estimated as a representation of the flow discontinuities. In $[6,8]$, the interaction consists in the cooperation between a dense flow field and a region-wise parametric polynomial flow. The dense optical flow is only encouraged to have some similarity with a piecewise parametric motion field associated to the partition map. In this paper, we present a motion estimation-segmentation method belonging to this latter class. We aim at developping a tight cooperation between a dense optic-flow estimator and a motion based segmentation process.

\section{Robust estimation of the optical flow}

Many standard optical flow estimators are based on the well known optical flow constraint (OFC) equation [13]. This differential equation, issued from a linearization of the brightness constancy assumption, links the spatio-temporal gradients of the luminance to the unknown velocity vector. In order to recover the two components of the velocity vector, a smoothness prior on the solution is usually introduced through an additional regularization term [5,13].

Due to the differential nature of the OFC, this standard modeling does not hold for large displacements. To circumvent the problem, it is usual to consider an incremental estimation of the flow field using a multiresolution setup [5, 11]. This multiresolution framework involving a pyramidal decomposition of the image data is standard and won't be emphasized herein. In the following, we shall assume to be working at a given resolution of this structure. However, one has to keep in mind that the expressions and computations are meant to be reproduced at each resolution level according to a coarse-to-fine strategy. Let us now assume that a rough estimate $\boldsymbol{w}=\left\{\boldsymbol{w}_{s}, s \in S\right\}$ of the unknown velocity field is available (e.g., from an estimation at lower resolution or from a previous estimation), on the rectangular pixel lattice $S$. Let $f(t)=\{f(s, t), s \in S\}$ be the luminance function at time $t$. Under the constancy brightness assumption from time $t$ to $t+1$, a small increment field $\mathrm{d} w \in \Omega \subset(\mathbb{R} \times \mathbb{R})^{S}$ can be estimated by minimizing the functional $\mathcal{H} \triangleq \mathcal{H}_{1}+\alpha \mathcal{H}_{2}$, with [5]:

$$
\begin{aligned}
& \mathcal{H}_{1}(\mathrm{~d} w) \triangleq \sum_{s \in S} \rho_{1}\left[\nabla f\left(s+w_{s}, t+1\right)^{T} \mathrm{~d} w_{s}+f_{t}\left(s, t, w_{s}\right)\right], \\
& \mathcal{H}_{2}(\mathrm{~d} w) \triangleq \sum_{<s, r>\in \mathcal{C}} \rho_{2}\left[\left\|\left(w_{s}+\mathrm{d} w_{s}\right)-\left(w_{r}+\mathrm{d} w_{r}\right)\right\|\right],
\end{aligned}
$$

where $\alpha>0, \mathcal{C}$ is the set of neighboring site pairs lying on grid $S$ equipped with some neighborhood system $\nu, \nabla f$ stands for the spatial gradient of $f$, $f_{t}\left(s, t, \boldsymbol{w}_{s}\right) \triangleq f\left(s+\boldsymbol{w}_{s}, t+1\right)-f(s, t)$ is the displaced frame difference, and functions $\rho_{1}$ and $\rho_{2}$ are standard robust $M$-estimators (with hyper-parameters $\sigma_{1}$ and $\sigma_{2}$ ). Functions $\rho_{1}$ and $\rho_{2}$ penalize both the deviations from the data model (i.e., the OFC) and from the first order smoothing prior. 
A robust $M$-estimator $\rho$ is an increasing cost function which compared to quadratic function possesses a saturating property $\left(\lim _{u \rightarrow \infty} \frac{\rho^{\prime}(u)}{2 u}=0\right)$. It allows to atenuate the influence of large "residual" values $[9,12]$. It can be shown that under certain simple conditions (mainly concavity of $\phi(v) \triangleq \rho(\sqrt{v})$ ), any multidimensional minimization problem of the form "find $\arg \min _{x} \sum_{i} \rho\left[g_{i}(x)\right]$ " can be turned into a dual minimization problem "find $\arg \min _{x, z} \sum_{i}\left[\tau z_{i} g_{i}(x)^{2}+\psi\left(z_{i}\right)\right]$ " involving auxiliary variables (or weights) $z_{i}$ s continuously lying in $(0,1]$ and $\tau$ is a parameter defined as $\tau \triangleq \lim _{v \rightarrow 0+} \phi^{\prime}(v)$. The function $\psi$ is a decreasing function depending on $\rho$ [9].

The new minimization is then usually led alternatively with respect to $x$ and to the $z_{i}$ s. If $g_{i}$ s are affine forms, minimization w.r.t. $x$ is a standard weighted least squares problem. In turn $x$ being frozen, the best weights have the following closed form:

$$
\hat{z}_{i}(x)=\frac{\rho^{\prime}\left[g_{i}(x)\right]}{2 \tau g_{i}(x)}=\frac{1}{\tau} \phi^{\prime}\left[g_{i}(x)^{2}\right] .
$$

In our case the weights are of two natures: (a) data outliers weights (related to the dual formulation of $H_{1}$ ), and (b) discontinuity weights (provided by the dual formulation of $H_{2}$ ) lying on the dual edge grid of $S$. The first set of weights, denoted by $\delta=\left\{\delta_{s}, s \in S\right\}$, allows to attenuate the effect of data for which the OFC is violated. The second one, denoted by $\beta=\left\{\beta_{s r},\langle s, r>\in \mathcal{C}\}\right.$, prevents from over-smoothing in locations exhibiting significant velocity discontinuities. The estimation is now expressed as the global minimization in $(\mathrm{d} w, \delta, \beta)$ of $\widetilde{\mathcal{H}} \triangleq$ $\widetilde{\mathcal{H}}_{1}+\alpha \widetilde{\mathcal{H}}_{2}$ where:

$$
\begin{aligned}
& \tilde{\mathcal{H}}_{1}(\mathrm{~d} \boldsymbol{w}, \delta)=\sum_{s \in S}\left[\tau_{1} \delta_{s}\left[\nabla f\left(s+\boldsymbol{w}_{s}, t+1\right)^{T} \mathrm{~d} \boldsymbol{w}_{s}+f_{t}\left(s, t, \boldsymbol{w}_{s}\right)\right]^{2}+\psi_{1}\left(\delta_{s}\right)\right], \\
& \widetilde{\mathcal{H}}_{2}(\mathrm{~d} \boldsymbol{w}, \beta)=\sum_{<s, r>\in \mathcal{C}}\left[\tau_{2} \beta_{s r}\left\|\left(\boldsymbol{w}_{s}+\mathrm{d} \boldsymbol{w}_{s}\right)-\left(\boldsymbol{w}_{r}+\mathrm{d} \boldsymbol{w}_{r}\right)\right\|^{2}+\psi_{2}\left(\beta_{s r}\right)\right] .
\end{aligned}
$$

More satisfactory robust cost functions being non-convex we end up with a difficult minimization problem to solved. We tackle it using a multigrid strategy that will be briefly described in section 4 . Let's first see how this energy function may be coupled with a segmentation device.

\section{Motion estimation and segmentation coupling}

We now introduce an extension of the model to couple the estimation process with a motion-based partition of the image. Let $\mathcal{P}$ denotes this partition composed of an unknown number $p$ of connected regions, $\mathcal{P}=\left\{\mathcal{R}_{1}, \ldots, \mathcal{R}_{i}, \ldots, \mathcal{R}_{p}\right\}$. The boundary between regions $\mathcal{R}_{i}$ and $\mathcal{R}_{j}$ will be noted $\partial_{i, j}$ and is defined as the set of edge-sites (i.e., lying on the dual edge grid) between $\mathcal{R}_{i}$ and $\mathcal{R}_{j}$ : $\partial_{i, j}=\left\{\langle s, r\rangle \in \mathcal{C}: s \in \mathcal{R}_{i}, r \in \mathcal{R}_{j}\right\}$. The set $\partial \mathcal{P} \triangleq \cup_{i \neq j} \partial_{i, j}$ stands therefore for the frontiers of the partition. 
The extension of the energy-based optic flow estimation model is obtained by incorporating two terms to the global energy function $\mathcal{H}^{1}$. The first one, $E_{\text {prior }}$, captures the a priori knowledge about the segmentation configuration. The second one, $E_{\text {inter }}$, specifies the mode of interaction between the segmentation and the rest of the estimation model (i.e., velocity field, weights and data).

We have chosen an interaction model which allows an interaction with the velocity field both at the frontiers and inside the regions: the segments will interact with the estimation process through the discontinuity weights, and through a parametric similarity of the motion profile inside each region.

The corresponding cost function component $E_{\text {inter }}(\mathcal{P}, \beta, \mathrm{d} w)$ exhibits two terms: The first one is proportional to the sum of $\beta_{s r}$ mean values over sets $\partial_{i, j}$. It then (a) favors low values (close to zero) of discontinuity weights along the borders and (b) guides the partition boundaries toward the most significant flow discontinuities. The second term enforces a polynomial parametric likeness of the flow field inside each region. The global energy of the extended model is designed as follows:

$$
\mathbb{H}(\mathrm{d} w, \mathcal{P}, \delta, \beta) \triangleq \widetilde{\mathcal{H}}(\mathrm{d} w, \delta, \beta)+E_{\text {prior }}(\partial \mathcal{P})+E_{\text {inter }}(\mathrm{d} w, \mathcal{P}, \beta),
$$

where,

$$
E_{\text {inter }}(\mathrm{d} \boldsymbol{w}, \mathcal{P}, \beta) \triangleq \mu_{1} \sum_{\partial_{i, j} \in \partial \mathcal{P}} \frac{1}{\left|\partial_{i, j}\right|} \sum_{<s, r>\in \partial_{i, j}} \beta_{s r}+\mu_{2} \sum_{\mathcal{R}_{i} \in \mathcal{P}} \sum_{s \in \mathcal{R}_{i}} \rho_{3}\left(\left\|\boldsymbol{w}_{s}+\mathrm{d} \boldsymbol{w}_{s}-\boldsymbol{w}_{s}^{\Theta_{i}}\right\|\right)
$$

with some positive parameters $\mu_{1}$ and $\mu_{2}$ and the function $\rho_{3}$ being a robust $M$ estimator with hyper-parameter $\sigma_{3}$. The vector $\boldsymbol{w}_{s}^{\Theta_{i}}$ is the parametric motion model associated to region $\mathcal{R}_{i}$. In this work, we will consider only the standard six parameter affine model: $\Theta=[a, b, c, d, e, f]^{T}$ with $\boldsymbol{w}_{s}^{\Theta_{i}}=P_{s} \Theta_{i}$, where $P_{s} \triangleq$ $\left(\begin{array}{cccccc}1 & x_{s} & y_{s} & 0 & 0 & 0 \\ 0 & 0 & 0 & 1 & x_{s} & y_{s}\end{array}\right)$. This model is usually considered as a good trade-off between model complexity and model efficiency [7]. It has been extensively used in motion analysis. This model conjectures that a given motion region is a projection of a 3D planar patch of surface whose motion is confined to rotations around the optical axis and to translations in its facet plan. According to $\S 2$ the interaction term may be rewritten in its dual form as:

$$
\begin{aligned}
\widetilde{E}_{\text {inter }}(\mathcal{P}, \beta, \mathrm{d} \boldsymbol{w}, \eta)= & \mu_{1} \sum_{\partial_{i, j} \in \partial \mathcal{P}} \frac{1}{\left|\mathcal{C}_{\partial_{i, j}}\right|} \sum_{<s, r>\in \partial_{i, j}} \beta_{s r}+ \\
& \mu_{2} \sum_{\mathcal{R}_{i} \in \mathcal{P}} \sum_{s \in \mathcal{R}_{i}} \tau_{3} \eta_{s}\left\|\boldsymbol{w}_{s}+\mathrm{d} \boldsymbol{w}_{s}-\boldsymbol{w}_{s}^{\Theta_{i}}\right\|^{2}+\psi_{3}\left(\eta_{s}\right) .
\end{aligned}
$$

The variables $\eta$ are auxiliary variables that we will refer to as parametric likeness weights throughout the paper. The resulting global energy is $\widetilde{\mathbb{H}}(\mathrm{d} w, \mathcal{P}, \delta, \beta, \eta)$.

${ }^{1}$ Even though the superscript remains omitted, we still suppose in the coming developments that some resolution level is concerned. 
The segmentation a priori term corresponds to a loose geometric constraint in terms of a classical Minimum Description Length (MDL) prior [14].

$$
E_{\text {prior }}(\partial \mathcal{P}) \triangleq \lambda|\partial \mathcal{P}| \text { for some } \lambda>0
$$

This energy term favors region with short and smooth border.

The whole energy function has now to be minimized with respect to all the unknowns. A direct minimization of such function is obviously a very intricate problem. As we shall see in the coming section, the optimization may be efficiently conducted through a multigrid minimization strategy.

\section{Multigrid optimization}

To efficiently cope with the optimization problem w.r.t. dw and $\mathcal{P}$, we design a joint hierarchical "constrained" exploration of the increment configuration space $\Omega$ and of the partition configuration space $\Upsilon$.

The overall optimization is led through a sequence of nested joint configuration subspaces:

$$
\left(\Omega^{L} \times \Upsilon^{L}\right) \subset\left(\Omega^{L-1} \times \Upsilon^{L-1}\right) \subset \cdots \subset\left(\Omega^{1} \times \Upsilon^{1}\right) \subset(\Omega \times \Upsilon)
$$

The subspace $\Omega^{\ell}$ is defined as the set of increment fields $\mathrm{d} w$ which are piecewise constant according to a $2^{\ell} \times 2^{\ell}$-block partition of grid $S^{2}$. Denote $\mathcal{B}^{\ell} \triangleq\left\{\mathcal{B}_{n}^{\ell}, n=\right.$ $\left.1 \ldots N_{\ell}\right\}$ this partition, the number of blocks being $N_{\ell}=|S| / 4^{\ell}$.

Each constrained configuration of $\Omega^{\ell}$ is "equivalent" to a reduced increment field $\mathrm{d} \boldsymbol{w}^{\ell}$ lying on the grid $S^{\ell} \triangleq\left\{1, \ldots, N_{\ell}\right\}$ associated with $\mathcal{B}^{\ell}$. Let $\Gamma^{\ell}$ be the set of such reduced configurations and let $\Phi_{1}^{\ell}$ be the point-to-point mapping from $\Gamma^{\ell}$ into $\Omega^{\ell}$.

In the same way, the partition configuration subspace $\gamma^{\ell}$ is the set of "constrained" partition $\mathcal{P}$ lying on the grid $S^{\ell}$ (i.e., defined on a $2^{\ell} \times 2^{\ell}$-block partition). Following the same methodology, a point-to-point mapping from a reduced partition configuration space $\Lambda^{\ell}$ into $\Upsilon^{\ell}$ may be easily constructed. Let $\Phi_{2}^{\ell}$ denote this function.

Constrained optimization in $\Omega^{\ell} \times \Upsilon^{\ell}$ is then equivalent to the minimization of the new energy function:

$$
\widetilde{\mathbb{H}}^{\ell}\left(\mathrm{d} w^{\ell}, \mathcal{P}^{\ell}, \delta, \beta, \eta\right) \triangleq \widetilde{\mathbb{H}}\left(\Phi_{1}^{\ell}\left(\mathrm{d} w^{\ell}\right), \Phi_{2}^{\ell}\left(\mathcal{P}^{\ell}\right), \delta, \beta, \eta\right)
$$

defined over $\Gamma^{\ell} \times \Lambda^{\ell}$, whereas the auxiliary variables, the data, and the field to be refined remain the same (i.e., defined on the original grid $S$ ).

At each resolution, we now have a cascade of optimization problems of reduced complexity:

$$
\underset{\mathrm{d} \boldsymbol{w}^{\ell}, \mathcal{P}^{\ell}, \delta, \beta, \eta}{\arg } \min \widetilde{\mathbb{H}}^{\ell}\left(\mathrm{d} \boldsymbol{w}^{\ell}, \mathcal{P}^{\ell}, \delta, \beta, \eta\right), \ell=L \ldots 0
$$

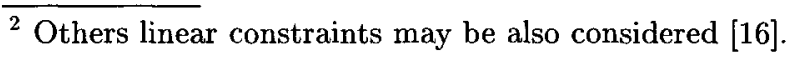


where $\mathrm{d} \boldsymbol{w}^{\ell} \in \Gamma^{\ell}$ and $\mathcal{P}^{\ell} \in \Lambda^{\ell}$ are defined on the reduced grid $S^{\ell}$, and auxiliary variables are attached to $S$, whatever the grid level.

This cascade of minimization problems is processed in terms of iteratively reweighted least squares within a multigrid coarse-to-fine strategy: the final estimates at level $\ell+1$ have a natural image at level $\ell$ (through $\left[\Phi_{1}^{\ell}\right]^{-1} \circ \Phi_{1}^{\ell+1}$ and $\left.\left[\Phi_{2}^{\ell}\right]^{-1} \circ \Phi_{2}^{\ell+1}\right)$, which are used as an initial configuration at level $\ell$.

\subsection{Multigrid energy derivation}

We now go into deeper details about the new multigrid function $\widetilde{\mathbb{H}}^{\ell}$ which is obviously composed of four terms similar to those of $\widetilde{\mathbb{H}}: \widetilde{\mathbb{H}}^{\ell}=\widetilde{\mathcal{H}}_{1}^{\ell}+\alpha \widetilde{\mathcal{H}}_{2}^{\ell}+$ $\widetilde{E}_{\text {inter }}^{\ell}+\widetilde{E}_{\text {prior }}^{\ell}$.

Data model adequation term: For any $n \in S^{\ell}$, denote $s_{1}, \ldots, s_{4^{\ell}}$ the sites of block $\mathcal{B}_{n}^{\ell}$, and define the following blockwise expressions:

$$
\begin{gathered}
\boldsymbol{\delta}_{n}^{\ell} \triangleq\left[\delta_{s_{1}} \cdots \delta_{s_{4^{\ell}}}\right]^{T}, \quad \Psi_{1}^{\ell}\left(\boldsymbol{\delta}_{n}^{\ell}\right) \triangleq \sum_{s \in \mathcal{B}_{n}^{\ell}} \psi_{1}\left(\delta_{s}\right), \quad \Delta_{n}^{\ell} \triangleq \operatorname{diag}\left(\delta_{s_{1}}, \ldots, \delta_{s_{4^{\ell}}}\right), \\
\boldsymbol{f}_{t}^{\ell}(n, \boldsymbol{w}) \triangleq\left[f_{t}\left(s_{1}, \boldsymbol{w}_{s_{1}}\right) \cdots f_{t}\left(s_{4^{\ell}}, \boldsymbol{w}_{s_{4^{\ell}}}\right)\right]^{T}, \nabla f^{\ell}(n, \boldsymbol{w}) \triangleq\left[\boldsymbol{f}_{x}^{\ell}(n, \boldsymbol{w}) \boldsymbol{f}_{y}^{\ell}(n, \boldsymbol{w})\right] \\
\boldsymbol{f}_{\bullet}^{\ell}(n, \boldsymbol{w}) \triangleq\left[f_{\bullet}\left(s_{1}+\boldsymbol{w}_{s_{1}}, t+1\right) \cdots f_{\bullet}\left(s_{4^{\ell}}+\boldsymbol{w}_{s_{4^{\ell}}}, t+1\right)\right]^{T}, \text { for } \bullet=x \text { or } y,
\end{gathered}
$$

Also, we will denote $\langle X \mid Y\rangle_{n} \triangleq X^{T} \Delta_{n}^{\ell} Y$ for any two $4^{\ell}$-row matrices or vectors, and $\|\boldsymbol{X}\|_{n}^{2} \triangleq\langle\boldsymbol{X} \mid \boldsymbol{X}\rangle_{n}$ for any $4^{\ell}$-component column vector. It is then easy to get the following compact expression:

$$
\widetilde{\mathcal{H}}_{1}^{\ell}\left(\mathrm{d} \boldsymbol{w}^{\ell}, \delta\right)=\sum_{n \in S^{\ell}}\left[\tau_{1}\left\|\nabla f^{\ell}(n, \boldsymbol{w}) \mathrm{d} \boldsymbol{w}_{n}^{\ell}+f_{t}^{\ell}(n, \boldsymbol{w})\right\|_{n}^{2}+\Psi_{1}^{\ell}\left(\delta_{n}^{\ell}\right)\right],
$$

which is very similar to the one of the "parent" energy $\widetilde{\mathcal{H}}_{1}(4)$. For each block, one gets a blockwise optical flow constraint expression involving aggregated observations.

Smoothing term: Let $\mathcal{C}_{n}^{\ell} \triangleq\left\{\langle s, r\rangle \in \mathcal{C}:\langle s, r\rangle \subset \mathcal{B}_{n}^{\ell}\right\}$ be the set of neighboring site pairs included in block $\mathcal{B}_{n}^{\ell}$ and $\mathcal{C}_{n m}^{\ell} \triangleq\left\{\langle s, r\rangle \in \mathcal{C}: s \in \mathcal{B}_{n}^{\ell}, r \in \mathcal{B}_{m}^{\ell}\right\}$ the set of neighboring site pairs straddling blocks $\mathcal{B}_{n}^{\ell}$ and $\mathcal{B}_{m}^{\ell}$. These sets $\left\{\mathcal{C}_{n}^{\ell}\right\}$ and $\left\{\mathcal{C}_{n m}^{\ell}\right\}$ form a partition of $\mathcal{C}$ and reduced grid $S^{\ell}$ turns out to be equipped with the same neighborhood system as $S$ (i.e., first- or second-order neighborhood system). The corresponding set of neighboring pairs will be denoted by $\mathcal{C}^{\ell}$. The smoothing term of $\widetilde{\mathcal{H}}^{\ell}$ is:

$$
\begin{aligned}
\tilde{\mathcal{H}}_{2}^{\ell}\left(\mathrm{d} \boldsymbol{w}^{\ell}, \beta\right)=\tau_{2}\left[\sum_{n \in S^{\ell}} \sum_{\left\langle s, r>\in \mathcal{C}_{n}^{\ell}\right.} \beta_{s r}\left\|\boldsymbol{w}_{s}-\boldsymbol{w}_{r}\right\|^{2}\right. \\
\left.\quad+\sum_{<n, m>\in \mathcal{C}^{\ell}} \sum_{\left\langle s, r>\in \mathcal{C}_{n m}^{\ell}\right.} \beta_{s r}\left\|\left(\boldsymbol{w}_{s}+\mathrm{d} \boldsymbol{w}_{n}^{\ell}\right)-\left(\boldsymbol{w}_{r}+\mathrm{d} \boldsymbol{w}_{m}^{\ell}\right)\right\|^{2}\right]+\sum_{<s, r>\in \mathcal{C}} \psi_{2}\left(\beta_{s r}\right)
\end{aligned}
$$


which reduces to:

$$
\mathcal{H}_{2}(\mathbf{0}, \beta)+\underset{<n, m>\in \mathcal{C}^{\ell}}{\tau_{2}}\left[\beta_{n m}^{\ell}\left\|\mathrm{d} \boldsymbol{w}_{n}^{\ell}-\mathrm{d} \boldsymbol{w}_{m}^{\ell}\right\|^{2}+2\left(\mathrm{~d} \boldsymbol{w}_{n}^{\ell}-\mathrm{d} \boldsymbol{w}_{m}^{\ell}\right)^{T} \overline{\Delta \boldsymbol{w}}_{n m}^{\ell}\right]
$$

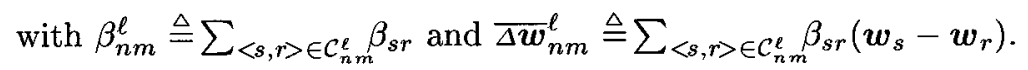

Parametric likeness term: Let us denote $\partial \mathcal{P}^{\ell}$ the border of partition $\mathcal{P}^{\ell}$ and let $\partial_{i, j}^{\ell} \triangleq \Phi_{2}^{\ell}\left(\partial_{i, j}\right)$ be the set of cliques straddling the frontiers resulting from the projection on $S$ of the edge $\partial_{i, j} \subset \partial P^{\ell}$ defined on $S^{\ell}$. We have:

$$
\begin{aligned}
\widetilde{E}_{\text {inter }}^{\ell}\left(\mathrm{d} w, \mathcal{P}^{\ell}, \beta, \eta\right)= & \mu_{1} \sum_{\partial_{i, j} \in \mathcal{P}^{\ell}} \frac{1}{\left|\partial_{i, j}^{\ell}\right|} \sum_{<s, r>\in \partial_{i, j}^{\ell}} \beta_{s r}+ \\
& \mu_{2} \sum_{\mathcal{R}_{i} \in \mathcal{P}^{\ell}} \sum_{n \in \mathcal{R}_{i}} \sum_{s \in \mathcal{B}_{n}^{\ell}} \eta_{s}\left\|\boldsymbol{w}_{s}+\mathrm{d} w_{n}^{\ell}-w_{s}^{\Theta_{i}}\right\|^{2}+\psi_{3}\left(\eta_{s}\right),
\end{aligned}
$$

which, like previously, reduces to:

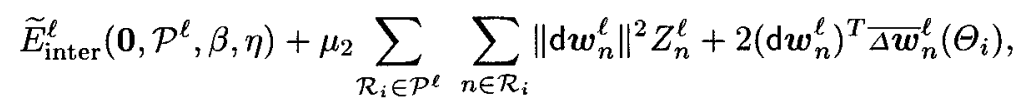

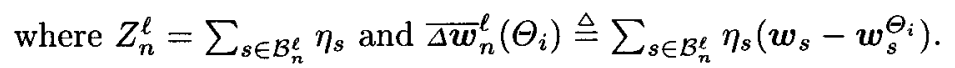

Partition a priori term: The corresponding expression of this term is straightforward. It is:

$$
E_{\text {prior }}^{\ell}\left(\partial \mathcal{P}^{\ell}\right)=2^{\ell} E_{\text {prior }}\left(\partial \mathcal{P}^{\ell}\right)=\lambda 2^{\ell}\left|\partial \mathcal{P}^{\ell}\right|
$$

\subsection{Energy minimization}

In this section we describe how the minimization of the total energy function is led. Due to the nature of the global function, we can devise an iteratively reweighted least squares minimization. The overall optimization process consists in an alternate minimization of the different weights and of the original variables of interest (namely $\mathrm{d} \boldsymbol{w}^{\ell}$ and $\mathcal{P}^{\ell}$ and the weights $\beta, \delta$, and $\eta$ ).

Let us first consider that the partition $\mathcal{P}^{\ell}$ and the parametric likeness weights are given.

Minimization w.r.t. the motion field and associated weights: We have thus to solve:

$$
\left(\widehat{\mathrm{d} w}^{\ell}, \hat{\beta}, \hat{\delta}\right)=\arg \min \widetilde{\mathcal{H}}_{1}^{\ell}+\alpha \widetilde{\mathcal{H}}_{2}^{\ell}+\widetilde{E}_{\text {inter }}^{\ell}
$$

This optimization is led in terms of iteratively reweighted least squares. The weights and the motion field are successively estimated in a recursive process 
until convergence. For a given set of weights the incremental field is computed from a weighted least squares estimation. The field being fixed the weights are then directly updated according to (3). To get a deeper insight in the considered minimization let us see exactly what are the updating rules under concern.

Consider that the motion $\mathrm{d} w^{\ell}$ is given. Let $\langle s, r\rangle$ be a clique in $\mathcal{C}$ and denote by $m$ and $n$ block numbers such that: $s \in \mathcal{B}_{n}^{\ell}$ and $r \in \mathcal{B}_{n}^{\ell}$. The optimal value of the discontinuity weights $\beta_{s r}$ is given respectively by:

- if the site pair is inside region $\mathcal{R}_{i}\left(<s, r>\subset R_{i}\right)$ :

$$
\hat{\beta}_{s r}=\frac{1}{\tau_{2}} \phi_{2}^{\prime}\left[\left\|\Delta w_{s r}^{\ell}\right\|^{2}\right]
$$

where, $\Delta \boldsymbol{w}_{s r}^{\ell} \triangleq\left(\boldsymbol{w}_{s}+\mathrm{d} \boldsymbol{w}_{n}^{\ell}\right)-\left(\boldsymbol{w}_{r}+\mathrm{d} \boldsymbol{w}_{m}^{\ell}\right)$ as a notational convenience.

- if the site pair straddles the "border" between regions $\mathcal{R}_{i}$ and $\mathcal{R}_{j}(<s, r>\epsilon$ $\left.\partial_{i, j}\right)$ :

$$
\hat{\beta}_{s r}=\frac{1}{\tau_{2}} \phi_{2}^{\prime}\left[\left\|\Delta w_{s r}^{\ell}\right\|^{2}+\frac{\mu_{1}}{\tau_{2}\left|\partial_{i, j}\right|}\right] .
$$

The data weights $\delta$ are only involved in $\widetilde{\mathcal{H}}_{1}$, therefore according to equation (3) and to the definition (1) of $\mathcal{H}_{1}$, the update rule is:

$$
\hat{\delta}_{s}=\phi_{2}^{\prime}\left[\nabla f\left(s+\boldsymbol{w}_{s}, t+1\right)^{T} \mathrm{~d} \boldsymbol{w}_{n}^{\ell}+f_{t}\left(s, t, \boldsymbol{w}_{s}\right)\right]
$$

Considering now that the weights $\beta$ and $\delta$ are frozen, the energy function $\widetilde{\mathbb{H}}$ is quadratic with respect to $\mathrm{d} \boldsymbol{w}^{\ell}$. Its minimization is equivalent to the resolution of a linear system:

$$
\left[\left\langle\nabla f^{\ell}(n, \boldsymbol{w})^{T} \mid \nabla f^{\ell}(n, \boldsymbol{w})\right\rangle_{n}+\gamma \mathbb{I}_{2}\right] \mathrm{d} \boldsymbol{w}_{n}^{\ell}+\left\langle\nabla f^{\ell}(n, \boldsymbol{w})^{T} \mid \boldsymbol{f}_{t}^{\ell}(n, \boldsymbol{w})\right\rangle_{n}-\gamma \overline{\boldsymbol{w}}_{n}^{\ell}=0
$$

with

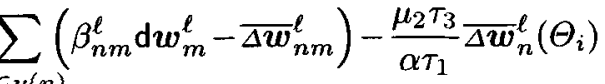

$$
\begin{aligned}
& \bar{w}_{n}^{\ell} \triangleq \frac{m_{m \in \nu(n)}}{\sum_{m \in \nu(n)} \beta_{n m}^{\ell}+\frac{\mu_{2} \tau_{3}}{\alpha \tau_{1}} Z_{n}^{\ell}}, \gamma \triangleq \frac{\alpha \tau_{2}}{\tau_{1}} \sum_{m \in \nu(n)} \beta_{n m}^{\ell}+\frac{\mu_{2} \tau_{3}}{\tau_{1}} Z_{n}^{\ell} \text {. }
\end{aligned}
$$

The solution of this linear system is searched using Gauss-Seidel relaxation method. Until convergence, every site $n$ of the grid $S^{\ell}$ is iteratively updated according to:

$$
\widehat{\mathrm{d} w}_{n}^{\ell}=\overline{\boldsymbol{w}}_{n}^{\ell}-\frac{\gamma\left\langle\nabla f^{\ell} \mid \nabla f^{\ell} \overline{\boldsymbol{w}}_{n}^{\ell}+\boldsymbol{f}_{t}^{\ell}\right\rangle_{n}+\operatorname{det} A \overline{\boldsymbol{w}}_{n}^{\ell}+\operatorname{com} A\left\langle\nabla f^{\ell} \mid \boldsymbol{f}_{t}^{\ell}\right\rangle_{n}}{\gamma(\gamma+\operatorname{trace} A)+\operatorname{det} A}
$$


with

$$
A \triangleq \nabla f^{\ell^{T}} \Delta_{n}^{\ell} \nabla f^{\ell}=\left[\begin{array}{cc}
\left\|f_{x}^{\ell}\right\|_{n}^{2} & \left\langle f_{x}^{\ell} \mid f_{y}^{\ell}\right\rangle_{n} \\
\left\langle f_{x}^{\ell} \mid f_{y}^{\ell}\right\rangle_{n} & \left\|f_{y}^{\ell}\right\|_{n}^{2}
\end{array}\right],
$$

and " $\operatorname{det} A$ ", "trace $A$ ", "com $A$ " stand respectively for the determinant, the trace and the cofactor matrix of $A$. Note that in the above expressions, $f_{x}^{\ell}, f_{y}^{\ell}, f_{t}^{\ell}$ vectors, and $\nabla f^{\ell}$ matrices as well, are displayed without $(n, \boldsymbol{w})$ for the sake of concision.

Minimization w.r.t. the partition and associated weights: Once the incremental motion field $\mathrm{d} \boldsymbol{w}^{\ell}$ and its associated weights are fixed, one has then to minimize $\widetilde{\mathbb{H}}$ w.r.t. the unknown partition $\mathcal{P}$ and the parametric likeness weights $\eta$, respectively. This optimization is conducted in the same alternate minimization spirit.

First the partition being fixed, the parametric weights and the motion parameters $\left(\Theta_{i}\right)$ are estimated through an iterated reweighted least squares estimation. For a given region $\mathcal{R}_{i} \in \mathcal{P}^{\ell}$, the update of the motion parameter vector is:

$$
\widehat{\Theta}_{i}=\left[\sum_{n \in \mathcal{R}_{i}} \sum_{s \in \mathcal{B}_{n}^{\ell}} \eta_{s}\left(P_{s}^{T} P_{s}\right)\right]^{-1} \sum_{n \in \mathcal{R}_{i}} \sum_{s \in \mathcal{B}_{n}^{\ell}} \eta_{s} P_{s}^{T}\left(w_{s}+\mathrm{d} w_{n}^{\ell}\right)
$$

while the parametric likeness updating rule is:

$$
\widehat{\eta}_{s}=\frac{1}{\tau_{3}} \phi_{3}^{\prime}\left(\left\|w_{s}+\mathrm{d} w_{n}^{\ell}-w_{s}^{\Theta_{i}}\right\|^{2}\right)
$$

Afterwards, the partition $\mathcal{P}^{\ell}$ has to be estimated. This estimation is done in two distinct steps: a local deformation step and a global deformation step. The first one consists in moving each point of the border $\partial \mathcal{P}$ within a small neighborhood in order to lower the associated energy terms. The second one consists in considering global transformations of the partition $\mathcal{P}$ such as appearance of new regions or merging of two adjacent regions. Let us carefully describe these two stages.

Local deformations: Iterative local deformations of the regions will be obtained by "moving" border sites of $\mathcal{P}$. This yields a new segmentation $\mathcal{P}$ '. Figure 4.2 shows an example of such deformations and settles the notational convention used in the following.

Let us compute the energy difference between two partitions $\mathcal{P}$ and $\mathcal{P}^{\prime}$. Let us denote $\Delta \partial \mathcal{P}^{\prime} \triangleq \partial \mathcal{P}^{\prime}-\partial \mathcal{P}$ the set of boundary pieces of partition $\mathcal{P}^{\prime}$ that were not included in $\partial \mathcal{P}$ and $\Delta \partial \mathcal{P} \triangleq \partial \mathcal{P}-\partial \mathcal{P}^{\prime}$ the set of border elements that have disappeared. Furthermore let us introduce the set composed of new portions of region created: $\Delta \mathcal{P}^{\prime} \triangleq\left\{\Delta \mathcal{R}_{i}^{\prime}, i=1 \ldots N\right\}$ (where $\Delta \mathcal{R}_{i}^{\prime}=\mathcal{R}_{i}^{\prime}-\mathcal{R}_{i}$ ) and the dual set of removed region portions $\Delta \mathcal{P} \triangleq\left\{\Delta \mathcal{R}_{i}, i=1 \ldots N\right\}$ (where 

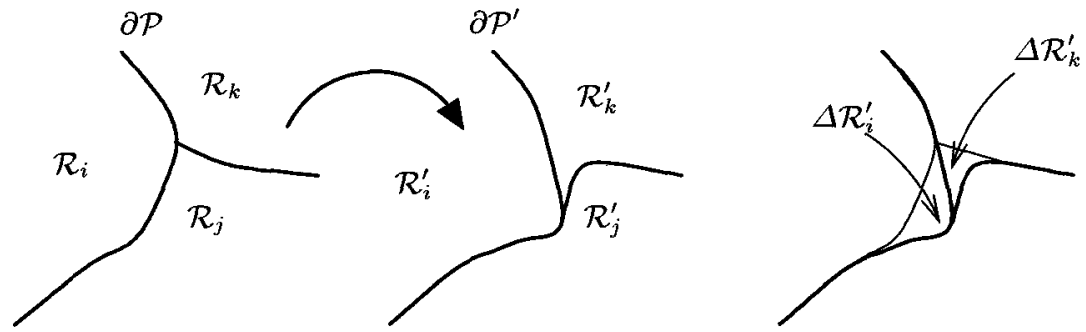

Fig. 1. Example of local deformations of the partition

$\left.\Delta \mathcal{R}_{i}=\mathcal{R}_{i}-\mathcal{R}_{i}^{\prime}\right)$. Assuming that region-wise motion parameters remain the same (since the deformation is only local) we have:

$$
\begin{aligned}
& \tilde{\mathbb{H}}^{\ell}\left(\mathcal{P}^{\prime \ell}\right)-\widetilde{\mathbb{H}}^{\ell}\left(\mathcal{P}^{\ell}\right)=E_{\text {prior }}\left(\Delta \partial \mathcal{P}^{\prime \ell}\right)-E_{\text {prior }}\left(\Delta \partial \mathcal{P}^{\ell}\right)+\sum_{\partial_{n, m} \in \Delta \partial \mathcal{P}^{\prime \ell}} \frac{\mu_{1}}{\left|\partial_{n, m}^{\ell}\right|} \sum_{<s, r>\in \partial_{n, m}^{\ell}} \beta_{s r}- \\
& \sum_{\partial_{n, m} \in \Delta \partial \mathcal{P}^{\ell}} \frac{\mu_{1}}{\left|\partial_{n, m}^{\ell}\right|} \sum_{<s, r>\in \partial_{n, m}^{\ell}} \beta_{s r}+\sum_{\Delta \mathcal{R}_{i}^{\prime} \in \Delta \mathcal{P}^{\ell}} \sum_{n \in \Delta \mathcal{R}_{i}^{\prime}} \sum_{s \in \mathcal{B}_{n}^{\ell}} \rho_{3}\left(\left\|w_{s}+\mathrm{d} w_{n}^{\ell}-w_{s}^{\Theta_{i}}\right\|\right)- \\
& \sum_{\Delta \mathcal{R}_{j} \in \Delta \mathcal{P}^{\ell}} \sum_{m \in \Delta \mathcal{R}_{j}} \sum_{s \in \mathcal{B}_{m}^{\ell}} \rho_{3}\left(\left\|w_{s}+\mathrm{d} w_{m}^{\ell}-w_{s}^{\Theta_{j}}\right\|\right) .
\end{aligned}
$$

The energy variation corresponding to a local deformation of the partition borders involves only local descriptors and is easily incrementally computed. In practice, a new position is considered for each border element of the current partition $\mathcal{P}^{\ell}$. If this position corresponds to an energy decrease it is accepted and the partition is updated. In all of our experiences, a border element is allowed to move one site forward or backward in the direction perpendicular to the border. Let us note that these displacements may be quite large ( $2^{\ell}$ sites) at coarse grid levels. However, we will suppose that our constancy assumption on motion parameters is still valid. This provides large computation time savings since the motion parameters do not have to be re-estimated.

Global deformations: The global deformations concern situations where the topology of the partition map is changed. This includes for example the appearing of new regions or the merging of adjacent regions. Other kinds of global transformations (e.g., rotations, scalings) could be also considered. In this work, only merges and appearance of new regions is considered.

The merging of two adjacent regions consists in removing their common boundary, when this yields a global energy decrease. Let $\mathcal{P}^{\prime} \triangleq \mathcal{P}-\left\{\mathcal{R}_{i}, \mathcal{R}_{j}\right\} \cup$ $\left\{\mathcal{R}_{i} \cup \mathcal{R}_{j}\right\}$ be obtained from $\mathcal{P}$ by the merging of $\mathcal{R}_{i}$ and $\mathcal{R}_{j}$. The difference of 
energy is $\widetilde{\mathbb{H}}^{\ell}(\mathcal{P})-\widetilde{\mathbb{H}}^{\ell}\left(\mathcal{P}^{\prime}\right)$ :

$$
\begin{gathered}
\sum_{\substack{\mathcal{R}_{k} \in \\
\mathcal{G}\left(\mathcal{R}_{i}\right) \cap \mathcal{G}\left(\mathcal{R}_{j}\right)}}\left[\left(\frac{\mu_{1}}{\left|\partial_{i, k}^{\ell} \cup \partial_{j, k}^{\ell}\right|}-\frac{\mu_{1}}{\left|\partial_{i, k}^{\ell}\right|}\right) \sum_{<s, r>\in \partial_{i, k}^{\ell}} \beta_{s r}+\left(\frac{\mu_{1}}{\left|\partial_{i, k}^{\ell} \cup \partial_{j, k}^{\ell}\right|}-\frac{\mu_{1}}{\left|\partial_{j, k}^{\ell}\right|}\right) \sum_{<s, r>\in \partial_{j, k}^{\ell}} \beta_{s r}\right]+ \\
\sum_{k \in\{i, j\}} \sum_{n \in \mathcal{R}_{k}} \sum_{s \in \mathcal{B}_{n}^{\ell}}\left[\rho_{3}\left(\left\|w_{s}+\mathrm{d} \boldsymbol{w}_{n}^{\ell}-\boldsymbol{w}_{s}^{\Theta_{i, j}}\right\|\right)-\rho_{3}\left(\left\|\boldsymbol{w}_{s}+\mathrm{d} \boldsymbol{w}_{n}^{\ell}-\right\| \boldsymbol{w}_{s}^{\Theta_{k}} \|\right)\right]-E_{\text {prior }}\left(\partial_{i, j}\right),
\end{gathered}
$$

where $\mathcal{G}\left(\mathcal{R}_{i}\right)$ denotes the set of adjacent regions of $\mathcal{R}_{i}-$ with $\mathcal{R}_{i} \notin \mathcal{G}\left(\mathcal{R}_{i}\right)$. This expression is evaluated for each pair of adjacent regions. The boundary leading to the greatest energy decrease is removed from the partition boundaries set and the corresponding regions are merged. This process is repeated until a complete stability is reached. Let us note that the motion parameter $\Theta_{i, j}$ corresponding to region $\mathcal{R}_{i} \cup \mathcal{R}_{j}$ has to be estimated only once (by iterated weighted least squares Equ. (25) and (26)), the first time the merging of the pair is evaluated.

The inclusion of a new region is evaluated according to a Markovian classification of the parametric likeness weights into two classes: $x \in\{0,1\}$. The first class gathers the outliers to the parametric likeness model whereas the second one groups points where the affine model fits well the dense velocity. A standard Markov random field model for supervised image classification is specified through the following energy (formulated here directly in the multigrid formalism):

$$
\hat{x^{\ell}}=\arg \min _{x^{\ell}} \sum_{n \in S^{\ell}} \sum_{s \in \mathcal{B}_{n}^{\ell}} \frac{1}{2 \sigma^{2}\left(x^{\ell}\right)}\left[m\left(x^{\ell}\right)-\eta_{s}\right]^{2}+2^{\ell+1}\left(2^{\ell}-\underset{<n, m>\in \mathcal{C}_{n, m}^{\ell}}{1) \lambda} \sum_{n} 1\left(x_{n}^{\ell}, x_{m}^{\ell}\right)\right.
$$

where $\mathbf{1}(x, y)=1$ if $x=y$ and $\mathbf{1}(x, y)=0$ otherwise. The parameters $\sigma(0)$ and $m(0)$ (respectively $\sigma(1)$ and $m(1)$ ) stand for the standard deviation and the mean of the outlier class (respectively the inlier class). In all the experiences these parameters have been fixed to the same set of values. If a sufficiently large region of outliers is detected by the classification process then it is included as a new region in the partition.

Global deformations obviously involve far more expensive computations than local deformations. Therefore, in practice, they will be only considered at the beginning of each grid level.

A sketchy synopsis of the overall method is presented in Fig. 2 within the multiresolution setup. In this figure, the subscript $k$ represents the resolution level. At the coarser resolution, the flow field is initialized to a null field associated to a partition composed of an unique region (the whole image). At that level, the partition is frozen in order to have a first crude estimate of the optical flow. At finer resolutions, the partition is initialized by a projection of previous segmentation on which global deformation process is run right away. 


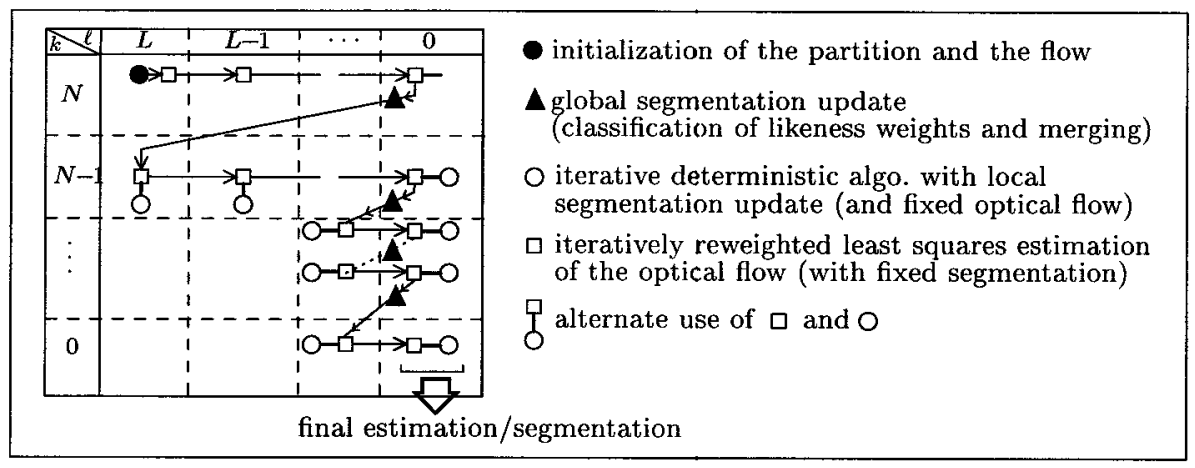

Fig. 2. Schematic synopsis of the complete estimation/segmentation method

\section{Experimental results}

The experiences have been carried out on synthetic sequence (for which a ground truth on the flow field to recover exists) and two real-world sequences. The first sequence is the well known Yosemite synthetic sequence (Fig. 3). The second test sequence is a Parking lot sequence which involves two cars moving in the foreground while the camera pans the scene. (Fig. 4). The last sequence, named Calendar includes several moving objects (a calendar moving vertically and a toy train pushing a ball) and an horizontal panning of the camera (Fig. 5).

As for the robust estimator, we choose the Leclerc's estimator $[14]: \rho(x) \triangleq$ $1-\exp \left(\frac{x^{2}}{\sigma^{2}}\right)$. Let us note that for each sequence, the same set of parameter values have been kept along the multiresolution setup and the multigrid structure. The number of resolution levels was respectively 2 for Calendar and Yosemite and 1 for Parking lot. The number of grid levels were fixed to 6 for Calendar and to 5 for the two others. The other parameters were tuned according to the amplitude of motion present in each sequence.

\begin{tabular}{|c|c|c|c|c|c|c|c|}
\hline Flow & $\bar{\mu}$ & $\boldsymbol{\sigma}$ & density & Technique & $\bar{\mu}$ & $\sigma$ & density \\
\hline Parametric flow & $4.54^{\circ}$ & $8.37^{\circ}$ & $100 \%$ & H. and S. (ori & $31.69^{\circ}$ & $31.18^{\circ}$ & $100 \%$ \\
\hline Dense flow & $4.91^{\circ}$ & $8.46^{\circ}$ & $100 \%$ & H. and S. (modified) & $9.78^{\circ}$ & $16.19^{\circ}$ & $100 \%$ \\
\hline A Estimation $/ \mathrm{s}$ & egmen & ation & nethod $\mathbf{\Lambda}$ & Uras et al. & \begin{tabular}{|l|}
$8.94^{\circ}$ \\
\end{tabular} & $15.61^{\circ}$ & $100 \%$ \\
\hline Dense flow [15] & $5.37^{\circ}$ & 8.19 & $100 \%$ & Lucas and Kanade & $4.28^{\circ}$ & $11.41^{\circ}$ & $35.1 \%$ \\
\hline$\Delta$ Optic-flow & estin & ation & $n e \Delta$ & Fleet and Jepson & $4.63^{\circ}$ & $13.42^{\circ}$ & $34.1 \%$ \\
\hline
\end{tabular}

Table 1. Comparative results on Yosemite

Following [4], we provide quantitative comparative results on Yosemite. Angular deviations with respect to the actual flow field have been computed. Tables above list the mean angular error $(\bar{\mu})$ and the associated standard deviation $(\sigma)$. They gather some of the results presented in [4] and those obtained by the estimation-segmentation method. In the latter case, we report the results for the parametric likeness flow and for the dense flow. Results obtained by the robust multigrid optical flow estimation method [15] without the segmentation coupling are also given. 
As may be seen from this table, our method provides almost as good results as those obtained with the best non-dense method. Compared with a single estimation of the optical flow the estimation-segmentation we propose improves the mean of the angular error. Furthermore, through the partition estimation we now have access to a global representation of the scene structure. Indeed, the obtained partition suggests a sensible planar patch representation of the underlying scene (Fig. 3). The parametric likeness field is actually a good approximation

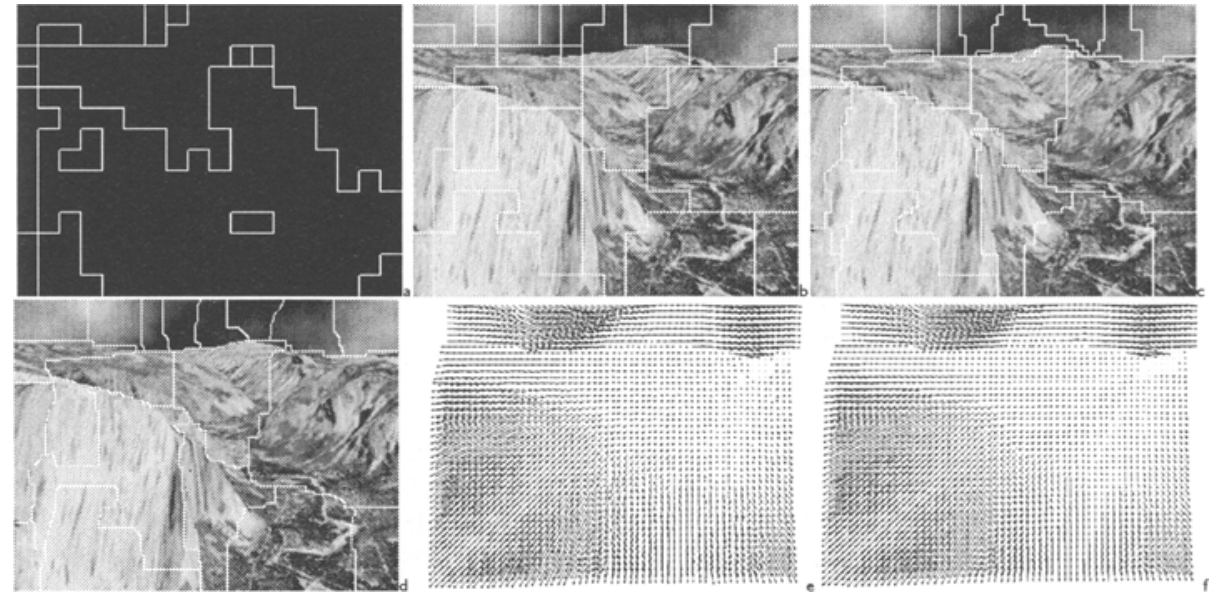

Fig. 3. Results on Yosemite $(224 \times 288)$ : (a) partition initialization at the coarsest level $\ell=4,(\mathbf{b}, \mathbf{c}, \mathbf{d})$ final partitions $(\ell=4,2,0)$, (e) dense optic flow, (f) parametric flow. (cpu-time 8mn.)

of the dense estimated flow field. This nice behavior of the method is confirmed by the results obtained on Calendar and Parking lot. For instance, in Calendar some difficult regions (such as the roof of the wagon or the space between the locomotive and the wagon) are fairly well recovered (Fig. 5). These remarks hold also in the Parking lot case where the method retrieves an interesting "structural approximation" of the front car (Fig. 4). We should also outline that the method is not very sensitive to the initialization. More precisely, the method is able to recover meaningful partitions of the flow field from quite "distant" initializations (see for instance Fig. 5 and Fig. 3).

Rough estimates of the computation times (code not hand-optimized) obtained on a Sun Ultra Sparc $(200 \mathrm{Mhz})$ are also given in the captions of figures 3,4 and 5 .

\section{Conclusion}

In this paper we have presented a method which relies on bilateral coupling between a dense optical flow estimator and a segmentation process. This approach is based on a global energy function where the interaction between the two processes lies both at the borders, through analog line process, and within each region through a parametric modeling of velocities.

The minimization of the global energy function is done using an efficient multigrid approach over both configuration spaces (dense velocity fields and 

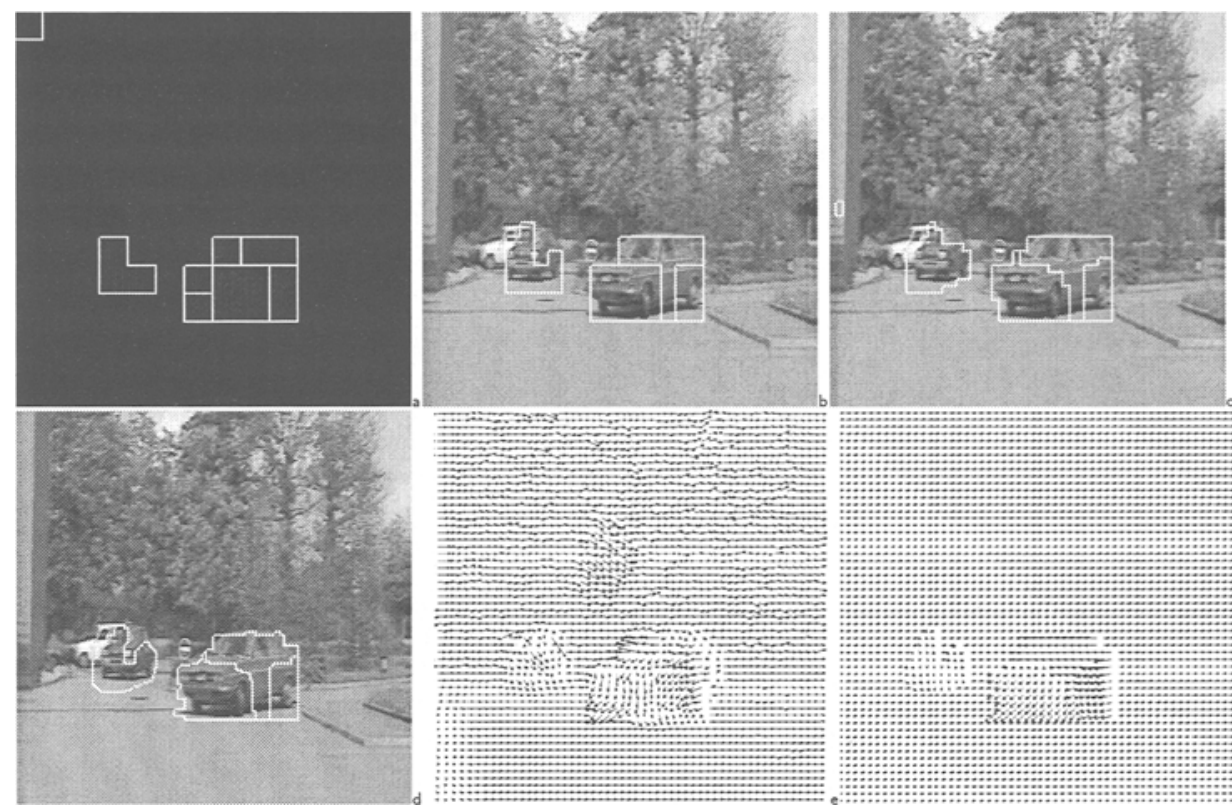

Fig. 4. Results on Parking lot $(224 \times 224)$ : (a) partition initialization at level $\ell=4$, (b, c, d) final partitions $(\ell=3,2,0)$, (e) dense optic flow, (f) parametric flow. (CPUTIME $4 m n$ 30s.)
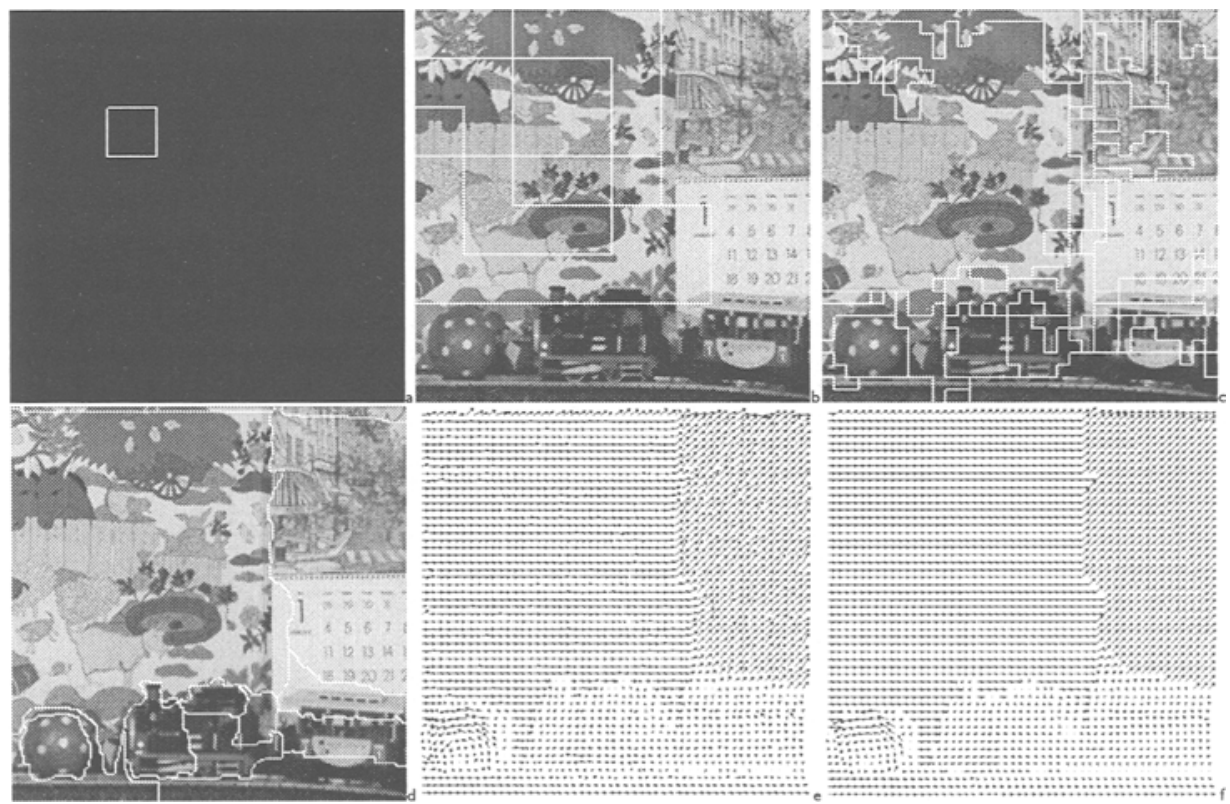

Fig. 5. Results on Calendar $(256 \times 256)$ : (a) partition initialization at level $\ell=4$, (b,c,d) final partitions $(\ell=5,3,0)$, (e) dense optic flow, (f) parametric flow. (CPUTIME 8mn.) 
image partitions). The overall minimization involving only incremental computation is computationally reasonable.

We have experimentally demonstrated that this coupling between a dense optical flow estimator and a segmentation process $(i)$ improves the global quality of the recovered dense flow field and (ii) provides a structural description of the entire motion field, and consequently of the underlying scene.

\section{References}

1. E.H. Adelson and J.Y.A. Wang. Representing moving images with layers. IEEE Trans. Pattern Anal. Machine Intell., 5(3):625-638, 1994.

2. G. Adiv. Determining three-dimensional motion and structure from optical flow generated by several moving objects. IEEE Trans. Pattern Anal. Machine Intell., 7:384-401, Jul 1985.

3. S. Ayer and H.S. Sawhney. Layered representation of motion video using robust maximum-likelihood estimation of mixture models and Mdl encoding. In Proc. Int. Conf. Computer Vision, pages 777-784, June 1995.

4. J. Barron, D. Fleet, and S. Beauchemin. Performance of optical flow techniques. Int. J. Computer Vision, 12(1):43-77, 1994.

5. M. Black and P. Anandan. The robust estimation of multiple motions: Parametric and piecewise-smooth flow fields. Computer Vision and Image Understanding, 63(1):75-104, 1996.

6. M. Black and P. Jepson. Estimating optical flow in segmented images using variable-order parametric models with local deformations. IEEE Trans. Pattern Anal. Machine Intell., 18(10):972-986, 1996.

7. P. Bouthemy and E. Francois. Motion segmentation and qualitative dynamic scene analysis from an image sequence. Int. J. Computer Vision, 10(2):157-182, 1993.

8. M. M. Chang, A. M. Tekalp, and M. I. Sezan. Simultaneous motion estimation and segmentation. IEEE Trans. Image Processing, 6(9):1326-1333, 1997.

9. P. Charbonnier, L. Blanc-Féraud, G. Aubert, and M. Barlaud. Deterministic edgepreserving regularization in computed imaging. IEEE Trans. Image Processing, 6(2):298-311, 1997.

10. 'T. Darrell and A.P. Pentland. Cooperative robust estimation using layers of support. IEEE Trans. Pattern Anal. Machine Intell., 17(5):474-487, 1995.

11. W. Enkelmann. Investigation of multigrid algorithms for the estimation of optical flow fields in image sequences. Comp. Vision Graph. and Image Proces., 43:150$177,1988$.

12. D. Geman and G. Reynolds. Constrained restoration and the recovery of discontinuities. IEEE Trans. Pattern Anal. Machine Intell., 14(3):367-383, 1992.

13. B. Horn and B. Schunck. Determining optical flow. Artificial Intelligence, 17:185$203,1981$.

14. Y. Leclerc. Constructing simple stable descriptions for image partitioning. Int. $J$. Computer Vision, 3:73-102, 1989.

15. E. Mémin and P. Pérez. Dense estimation and object-based segmentation of the optical flow with robust techniques. IEEE Trans. Image Processing, 5(1), 1998.

16. E. Mémin and P. Pérez. A multigrid approach for hierarchical motion estimation. In Proc. Int. Conf. Computer Vision, pages 933-938, 1998.

17. D.W. Murray and H. Buxton. Scene segmentation from visual motion using global optimization. IEEE Trans. Pattern Anal. Machine Intell., 9(2):220-228, Mar 1987.

18. C. Stiller. Object-based estimation of dense motion fields. IEEE Trans. Image Processing, 6(2):234-250, 1997. 